Letter

\title{
Safety of chitosan processed wine in shrimp allergic patients
}

Wine is an ubiquitous product all over the world, but up to 1 in 10 consumers reports intolerance or allergy-like symptoms after drinking wine. ${ }^{1}$ Chitosan is a linear polysaccharide and the product of the deacetylation of chitin, the main component of the cell walls of some fungi; the exoskeletons of arthropods, such as crustaceans and insects; and the beaks of cephalopods. ${ }^{2}$ It also may be used in wine processing.

Concerns have been raised that wines processed with chitosan could trigger reactions in patients with seafood allergy. Nevertheless, biological plausibility is scarce. Major allergens involved in shrimp allergy are muscle proteins, although some minor allergens, such as arginine kinase, which was found to be clinically relevant, have been found in shrimp shells. ${ }^{3}$ The chitosan isolation procedure is expected to remove all proteins and contaminants. Fear of ingesting a shellfish-derived product may lead to unnecessary avoidance of wine produced with chitosan films.

Recently, anaphylactic reaction to $\alpha$-1,3-galactose has been identified. ${ }^{4}$ Chitosan is structurally different from $\alpha$-gal because it is a linear cationic $(1 \rightarrow 4)$-2-amino-2-deoxy- $\beta$-D-glucan. There is only one case report of an immediate anaphylactic reaction with health food (fungiderived) chitosan ingestion, with documented sensitization by skin prick tests. ${ }^{5}$ Shrimp-derived chitosan has been given the classification of generally recognized as safe (GRAS) by the US Food and Drug Administration (FDA) in 2012. ${ }^{6}$ Moreover, evidence supporting this classification is limited by the lack of inclusion of individuals with shrimp allergy and the absence of food challenges. To our knowledge, no previous studies have evaluated the safety of chitosan as a food additive in shrimp allergic patients. Therefore, we aimed to assess the safety of chitosan processed wine in shrimp allergic patients.

This double-blind, placebo-controlled trial was registered at ClinicalTrials.gov (NCT02151279). Adults (18-65 years) with evidence of IgE-mediated sensitization to shrimp and a history of anaphylaxis to shrimp and/or a positive oral challenge result to shrimp were selected. Participants were recruited from the food allergy unit of an allergy department of a university hospital. A control group of 6 healthy, nonatopic, non-food allergic individuals were invited to participate. All individuals included in this study were regular consumers of wine and were asked to avoid alcohol ingestion for at least 3 days before the challenge. Nineteen individuals were enrolled, including 13 with anaphylaxis to shrimp (Table 1) and 6 healthy controls.

Skin prick-to-prick tests with wines were performed, as well as a double-blind, placebo-controlled challenge with both wines (with and without chitosan) in all participants. The challenges were performed on the same day, separated by at least 2 hours. Successive increasing doses were administered in 4 steps with 15 -minute intervals up to a total of $100 \mathrm{~mL}$ of each wine during each challenge. On completion, participants were observed for 2 hours, and, in case of any delayed reaction, participants were instructed to contact the

Disclosures: Authors have nothing to disclose. investigators. The research ethics committee, Comissão de Ética para a Saúde do Centro Hospitalar São João e Faculdade de Medicina da Universidade do Porto, approved the study, and signed informed consent was obtained from all participants.

The wines processed with chitosan-based films (active challenge) and the wines conserved with sulfur dioxide were indistinguishable in color, smell, or taste, at least for nonsommelier study participants. After fermentation, instead of using sulfur dioxide for wine preservation, chitosan films cross-linked with genipin were immersed in the wine in a proportion of $60 \mathrm{~cm}^{2} / \mathrm{L}$ of wine.

A positive skin test result was defined as the presence after 15 minutes of a wheal mean diameter of $3 \mathrm{~mm}$ or larger than that elicited by the negative control accompanied by erythema. Positive (histamine, $10 \mathrm{mg} / \mathrm{mL}$ ) and negative (saline solution) controls were also included. During challenges, occultation was performed by an ombudsman, and each wine was placed in identified equal containers. The randomization codes were packed in sealed, nontransparent envelopes that remained closed until the end of the study. Only 1 shrimp allergic participant had positive skin prick test result to both wines. All the other participants had negative skin test results to both wines. None of the 13 shrimp allergic patients and none of the control participants had immediate- or late-phase reactions to any of the wines. No subjective symptoms were reported; therefore, no medical intervention was necessary.

Our study found that wine processed with chitosan-based films as a preservative agent may be safely consumed by shrimp allergic individuals. We selected patients with severe shrimp allergy, and all of them tolerated this wine; consequently, it is unlikely that individuals with a similar history of allergic reactions or mild allergic reactions would react to this type of wine. To our knowledge, this is the first study to evaluate the safety of seafood-derived chitosan in shrimp allergic patients, using double-blind, placebo-controlled challenges.

We also found that the isolated use of skin testing is insufficient for diagnosis. One patient had positive skin test results to both wines. Skin prick testing with nonstandardized matrices, such as wine, can be associated with nonspecific testing, as previously described for orange juice. ${ }^{8}$ However, there was no clinical significance of the positive skin test results because the oral challenge result was negative. Another strength is that our sample only included patients with anaphylaxis to shrimp. This decreases the concern that reactions may occur only in a dose-dependent manner and reinforces the statement of the FDA that chitosan is GRAS, even in patients with severe shrimp allergy.

Chitosan is considered to be a nontoxic and nonallergenic material that is suitable for use in medical and pharmaceutical applications. ${ }^{9}$ Furthermore, it has been tested as topic bandages in shellfish allergic patients, and no adverse reactions were reported. ${ }^{10}$ Our study contributes to overcome the paucity of data regarding the patients with shrimp allergy. Furthermore, evidence that sulfite-free chitosan-treated wines are also a safe alternative for shrimp allergic patients broadens its use. 
Table 1

Demographic and clinical characteristics of the shrimp allergic patients

\begin{tabular}{lc}
\hline Characteristics & $\mathrm{N}(\%)$ \\
\hline Age (years), median IQR & $34,[26-42]$ \\
Female & $10(77)$ \\
House dust mite sensitization & $11(85)$ \\
Atopic diseases & $13(100)$ \\
$\quad$ Allergic rhinitis & $13(100)$ \\
Asthma & $5(38.5)$ \\
Atopic eczema & $2(15.4)$ \\
Symptoms & \\
Anaphylaxis & $13(100)$ \\
Cutaneous & $13(100)$ \\
Other & $13(100)$ \\
Time (years) since last shrimp allergic reaction, median IQR & $2,[1-4]$ \\
Wheal size of skin prick test with shrimp extract, mean (sd) & $6.1(2.1)$ \\
Wheal size of skin prick-prick test, mean (sd) & $9.4(7.5)$ \\
Shrimp slgE (kU/L), mean (sd) & $12.5(31.4)$ \\
\hline
\end{tabular}

Abbreviations: IQR, interquartile range; sd, standard deviation; sIgE, specific IgE.

Data is presented as number and percentage, except when otherwise stated.

${ }^{a}$ Dyspnea, throat tightness, oropharyngeal pruritus, hypotension, vomit, abdominal pain and diarrhea.

The number of included participants may be a limitation. Nonetheless, all the participants had previous history of anaphylaxis to shrimp and other seafood, and all of them had atopic comorbidities, making them a particularly high-risk group. In conclusion, this study indicates that wine treated with chitosan is unlikely to trigger allergic reactions in patients with shrimp allergy.

Luís Amaral, MD

Diana Silva, $\mathrm{MD}^{*}, \dagger$

Mariana Couto, $\mathrm{MD}^{\dagger,}$

Cláudia Nunes, PhD

Sílvia M. Rocha, PhD

Manuel A. Coimbra, PhD

Alice Coimbra, MD

André Moreira, MD, PhD*,
*Serviço de Imunoalergologia

Centro Hospitalar São João

${ }^{\dagger}$ Faculty of Medicine of University of Porto

${ }^{\ddagger}$ Allergy Unit

Hospital \& Instituto CUF Porto

Porto, Portugal

${ }^{\S}$ QOPNA \& Departamento de Química

Universidade de Aveiro

Aveiro, Portugal

luis.m.amaral@gmail.com

\section{References}

[1] Wigand P, Blettner M, Saloga J, Decker H. Prevalence of wine intolerance: results of a survey from Mainz. Germany. Dtsch Arztebl Int. 2012;109: 437-441.

[2] Muzzarelli RAA. Chitins and chitosans as immunoadjuvants and nonallergenic drug carriers. Mar Drugs. 2010;8:292-312.

[3] Khanaruksombat S, Srisomsap C, Chokchaichamnankit D, Punyarit P, Phiriyangkul P. Identification of a novel allergen from muscle and various organs in banana shrimp (Fenneropenaeus merguiensis). Ann Allergy Asthma Immunol. 2014;113:301-306.

[4] Steinke JW, Platts-Mills TA, Commins SP. The alpha-gal story: lessons learned from connecting the dots. JAllergy Clin Immunol. 2015;135: 589-596.

[5] Kato Y, Yagami A, Matsunaga K. A case of anaphylaxis caused by the health food chitosan [in Japanese]. Arerugi. 2005;54:1427-1429.

[6] US Food and Drug Administration. GRAS Notices: Shrimp-Derived Chitosan http://www.accessdata.fda.gov/scripts/fdcc/?set=GRASNotices\&id=443\&sort $=$ GRN_No\&order $=$ DESC\&startrow $=1 \&$ type $=$ basic\&search $=443 . \quad$ Accessed January 13, 2014

[7] Nunes C, Maricato É, Cunha Â, Nunes A, da Silva JAL, Coimbra MA. Chitosan-caffeic acid-genipin films presenting enhanced antioxidant activity and stability in acidic media. Carbohydr Polym. 2013;91:236-243.

[8] Brockow K, Hautmann C, Fötisch K, et al. Orange-induced skin lesions in patients with atopic eczema: evidence for a non-IgE-mediated mechanism. Acta Derm Venereol. 2003;83:44-81.

[9] Hayashi Y, Yamada S, Yanagi Guchi K, Koyama Z, Ikeda T. Chitosan and fish collagen as biomaterials for regenerative medicine. In: Se-Kwon K, ed. Advances in Food and Nutrition Research. Cambridge, MA: Academic Press; 2012: $107-120$.

[10] Waibel KH, Haney B, Moore M, Whisman B, Gomez R. Safety of chitosan bandages in shellfish allergic patients. Mil Med. 2011;176:1153-1156. 